\title{
Achievable Transmission Capacity of Cognitive Radio Networks with Cooperative Relaying
}

\author{
Xiuying Chen ${ }^{1}$, Tao Jing ${ }^{1}$, Yan $\mathrm{Huo}^{1}$, Wei $\mathrm{Li}^{2}$, Xiuzhen Cheng ${ }^{2}$, Tao Chen ${ }^{3}$ \\ ${ }^{1}$ School of Electronics and Information Engineering, Beijing Jiaotong University, Beijing, China \\ ${ }^{2}$ Computer Science, The George Washington University, Washington DC, USA \\ ${ }^{3}$ VTT - Technical Research Centre of Finland, Kaitoväylä 1, Oulu, Finland \\ $\{10120069$, tjing, yhuo\}@bjtu.edu.cn, \{weili,cheng\}@gwu.edu, tao.chen@vtt.fi
}

\begin{abstract}
Previous research on cognitive networks argues that secondary users can only work under a low transmission power condition in an underlay spectrum sharing model. Motivated by the idea of cooperative communications, in this paper we investigate the achievable transmission capacity of a cognitive network that provides cooperative relaying to the primary network over the underlay spectrum sharing model. The feasible region of the relay, the lower bound of the power ratio between the primary network and the secondary network with or without cooperative relaying, as well as the maximum achievable transmission capacity of the secondary network with or without relaying under the outage constraints from both the primary and the secondary network, are carefully studied. Numerical results indicate that secondary users can achieve a higher transmission capacity with cooperative relaying as they can transmit at a higher power while satisfying the outage probability constraints from both systems.
\end{abstract}

Index Terms-Achievable transmission capacity; cognitive radio networks; cooperative relay; outage probability

\section{INTRODUCTION}

As a fundamental problem, achievable transmission capacity of a cognitive radio network has been extensively studied $[1]-[6],[9]-[13]$. Our prior work [1] indicates that secondary users could only work at a low transmission power over the underlay spectrum sharing model to guarantee the normal communications of the primary users, especially when the direct transmission from a primary transmitter to its receiver is severely damaged due to pass loss and channel fading; thus secondary users can only achieve a low transmission capacity.

Motivated by the physical layer technology called cooperative relaying, in this paper we aim to study whether cooperative communications can help the secondary users to achieve a higher transmission capacity constrained by the outage probabilities from both the primary and the secondary system compared to the cognitive network without cooperative relaying under the physical interference model. Our work deviates from most existing works as they usually assume the mechanism in which primary users lease their spectrum to secondary users for a fraction of time and in exchange, they get cooperative transmissions or other benefits. In this paper, we investigate the achievable transmission capacity of a cognitive network that provides cooperative relaying to the primary network over the underlay spectrum sharing model, which has not been addressed to our best knowledge.
The cooperation between the primary and secondary system under the interleave spectrum sharing model has been investigated in [4], [5], which demonstrate that cognitive cooperation can support a higher stable throughput for both the primary and the secondary users compared to non-cooperative networks. A tradeoff on the utilities of the primary and the secondary users is studied in [6], and the results indicate that the primary and secondary users have the motivation to cooperate with each other under certain circumstances in which the performance of both systems can be dramatically improved if they cooperate.

Our main contributions are summarized as follows: 1) The successful transmission probabilities of the primary and the secondary network under the physical interference model are derived based on our cooperative network framework. 2) The maximum average transmission capacities of the cognitive network with/without cooperative relaying are derived under the outage constraints from both the primary and the secondary network when the decode-and-forward relaying protocol is adopted. 3) Numerical analysis is reported to verify our argument which states that cognitive networks can achieve a higher transmission capacity when secondary users provide cooperative relaying for the primary network over the underlay spectrum sharing model.

The rest of the paper is organized as follows. Section II depicts our system model. In Section III we derive the achievable transmission capacity of the secondary network when no cooperative relaying is employed. Section IV details the elaboration on the achievable transmission capacity with cooperative relays. Our numerical analysis is reported in Section $\mathrm{V}$ and Section VI concludes the paper.

\section{SySTEM MODEL}

\section{A. Network Model}

We consider a system model depicted in Fig. 1, where a primary (licensed) transmitter PT communicates with an intended primary receiver PR. In the same spectrum band, a secondary network, composed of $N$ nodes, resides in the range of the primary network and is seeking to exploit possible transmission opportunities. When the PT is far from the PR, a secondary user, which has a higher link quality and is not in transmitting or receiving mode, can be selected to relay packets for the PT. Such a SU is called a cooperative relay. For simplicity, we assume that the primary user employs a 


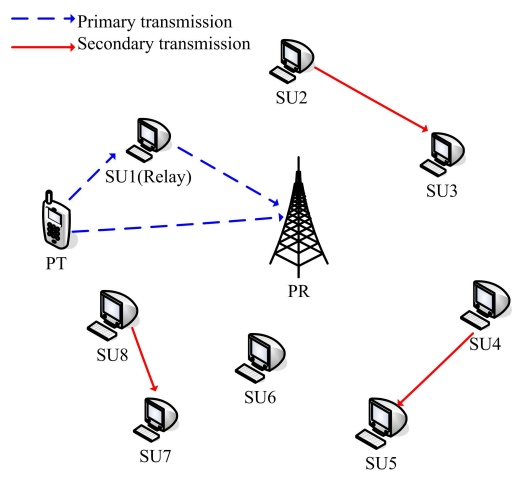

Fig. 1. The system model.

fixed transmission power $P_{p}$, and all secondary transmitters have the same transmission power $P_{s}$.

We further assume that time is slotted, and that the transmission of one packet for both the primary network and the secondary network takes the duration of exactly one time slot. When a cooperative relay is utilized, the delivery of a packet from the PT to the PR should take two time slots, with the first one for the transmission from the PT to the relay, and the second one from the relay to the PR. Thus we consider two successive time slots in our analysis. If no cooperative relay is employed, the PT transmits a packet to the PR directly in the first slot and is idle in the second slot. The secondary network can transmit at both slots, as long as the interference experienced by the PR is tolerable.

\section{B. Physical Layer Model}

For a propagation channel model with a long term path loss and a short term independent flat Rayleigh fading, the received power at a typical receiver from a transmitter can be computed by $P_{k} \delta_{i j}\left|d_{i j}\right|^{-\alpha}$, where $P_{k}$ is the transmission power of network $k$, with $k=p$ denoting the primary network and $k=s$ denoting the secondary network, $\alpha$ is the path loss exponent, $d_{i j}$ is the distance between the transmitting node $i$ and the receiver $j$, and $\delta_{i j}$ is the fading factor on the power transmitted from the node $i$ to the receiver $j$. Let $i=0$ denote the PT, and $j=0$ denote the PR. Then other values of $i$ and $j$ denote secondary users. The probability density function of the fading factor $\delta_{i j}$ follows an exponential distribution with a unit mean [8]. Considering the cumulative interference from the transmitters of both the primary network and the secondary network, the signal to interference-plus-noise ration (SINR) at the receiver $j$ of system $k$ can be represented by:

$$
S I N R_{i j}=\frac{P_{k} \delta_{i j} d_{i j}^{-\alpha}}{I_{p j}+I_{s j}+N_{0}}
$$

where $N_{0}$ is the thermal noise power, and $I_{p j}=P_{p} \delta_{0 j}\left|d_{0 j}\right|^{-\alpha}$ and $I_{s j}=\sum_{q \in S U} P_{s} \delta_{q j}\left|d_{q j}\right|^{-\alpha}$ (for $q \neq i$ ) are respectively the cumulative interference power from the transmitting node of the primary network and that of the secondary network to the typical receiver $j$ of network $k$. Note that $I_{p j}=0$ when $j=0$. As spectrum sharing systems are interference-limited [9], the thermal noise can be negligible. Hence for simplicity, SIR is used instead of SINR:

$$
S I R_{i j}=\frac{P_{k} \delta_{i j} d_{i j}^{-\alpha}}{I_{p j}+I_{s j}}
$$

A signal can be correctly decoded at a receiver of system $k$ if the corresponding SIR is greater than a threshold $\eta_{k}$. Thus the probability of a successful transmission can be defined as $\operatorname{Pr}\left(S I R_{i j} \geq \eta_{k}\right)$.

\section{Achievable Transmission Capacity}

Since the achievable transmission capacity in packets/s/node does not take into account the spectral efficiency of each packet, we define the transmission capacity in bits/s/Hz/node, which measures the number of bits each node can receive from its desired transmitter per second per Hertz. A similar argument can be found in [7]. According to Shannon's Theory, a packet can carry $\log _{2}\left(1+\eta_{s}\right)$ bits/s/Hz information. Thus the achievable transmission capacity can be defined as:

$$
C=\log _{2}\left(1+\eta_{s}\right) \operatorname{Pr}\left(S I R_{i j} \geq \eta_{s}\right)
$$

\section{ACHIEvable Transmission CAPACITy Without COOPERATIVE RELAYING}

As a baseline, we first analyze the achievable transmission capacity of the secondary network when no cooperative relay is utilized. In such a case, the PT transmits signals to its PR directly. Assume that a subset of SUs, denoted by Sub, are allowed to transmit over the same spectrum band as the PT in each time slot, as long as their transmissions do not disturb the normal communications of the primary network.

According to (2), the successful transmission probability of the PT in the first slot can be given as:

$$
\begin{aligned}
& \operatorname{Pr}\left(S I R_{00} \geq \eta_{p}\right)=\operatorname{Pr}\left(\frac{P_{p} \delta_{00} d_{00}^{-\alpha}}{I_{s 0}} \geq \eta_{p}\right)=\operatorname{Pr}\left\{\delta_{00} \geq \frac{\eta_{p} d_{00}^{\alpha}}{P_{p}} I_{s 0}\right\} \\
& \quad=\mathrm{E}_{\left\{\delta_{i 0}\right\}}\left\{\exp \left(-\frac{\eta_{p} d_{00}^{\alpha}}{P_{p}} \sum_{i \in\{S u b\}} P_{s} \delta_{i 0} d_{i 0}^{-\alpha}\right)\right\} \\
& \quad=\prod_{i \in\{\text { Sub }\}} \frac{1}{1+\frac{\eta_{p}}{\gamma_{p s}}\left(\frac{d_{00}}{d_{i 0}}\right)^{\alpha}}
\end{aligned}
$$

where $\gamma_{p s}=\frac{P_{p}}{P_{s}}$ is the power ratio between the primary network and the secondary network.

Similarly, the successful transmission probability of a secondary user in the first time slot is given by:

$$
\begin{aligned}
\operatorname{Pr}_{f i r s t}\left(S I R_{i j} \geq \eta_{s}\right)=\operatorname{Pr}\left(\frac{P_{s} \delta_{i j} d_{i j}^{-\alpha}}{I_{s j}+I_{p j}} \geq \eta_{s}\right) \\
=\exp \left(-\frac{\eta_{s} d_{i j}^{\alpha}}{P_{s}}\left(I_{s j}+I_{p j}\right)\right) \\
=\mathrm{E}_{\left\{\delta_{q j}\right\}}\left\{\exp \left(-\frac{\eta_{s} d_{i j}^{\alpha}}{P_{s}} \sum_{q \in\{S u b \backslash i\}} P_{s} \delta_{q j} d_{q j}^{-\alpha}\right)\right\} \\
\quad \times \mathrm{E}_{\left\{\delta_{0 j}\right\}} \exp \left(-\frac{\eta_{s} d_{i j}^{\alpha}}{P_{s}} P_{p} d_{0 j}^{-\alpha} \delta_{0 j}\right) \\
=\prod_{q \in\{S u b \backslash i\}} \frac{1}{1+\eta_{s}\left(\frac{d_{i j}}{d_{q j}}\right)^{\alpha}} \frac{1}{1+\eta_{s} \gamma_{p s}\left(\frac{d_{i j}}{d_{0 j}}\right)^{\alpha}}
\end{aligned}
$$


Based on (3), the achievable transmission capacity of the secondary node in the first slot can be computed by:

$$
C_{\text {first }}=\log \left(1+\eta_{s}\right) \prod_{q \in\{S u b \backslash i\}} \frac{1}{1+\eta_{s}\left(\frac{d_{i j}}{d_{q j}}\right)^{\alpha}} \frac{1}{1+\eta_{s} \gamma_{p s}\left(\frac{d_{i j}}{d_{0 j}}\right)^{\alpha}}
$$

As the PR can receive its packet successfully in the first time slot, PT stays idle during the second slot, leaving the opportunity for the secondary nodes to access the spectrum without disturbing the primary network. Hence, the successful transmission probability of a secondary user in the second slot can be expressed by:

$$
\operatorname{Pr}_{\text {second }}\left(S I R_{i j} \geq \eta_{s}\right)=\prod_{q \in\{\text { Sub } \backslash i\}} \frac{1}{1+\eta_{s}\left(\frac{d_{i j}}{d_{q j}}\right)^{\alpha}}
$$

Accordingly, the achievable transmission capacity of the secondary node can be given by:

$$
C_{\text {second }}=\log \left(1+\eta_{s}\right) \prod_{q \in\{\text { Sub } \backslash i\}} \frac{1}{1+\eta_{s}\left(\frac{d_{i j}}{d_{q j}}\right)^{\alpha}}
$$

From (4) (8), we can obtain the following average transmission capacity of a secondary user with outage constraints from both the primary and the secondary network:

$$
C=\frac{1}{2} \log \left(1+\eta_{s}\right) \prod_{q \in\{S u b \backslash i\}} \frac{1}{1+\eta_{s}\left(\frac{d_{i j}}{d_{q j}}\right)^{\alpha}}\left[\frac{1}{\eta_{s} \gamma_{p s}\left(\frac{d_{i j}}{d_{0 j}}\right)^{\alpha}}+1\right]
$$

subject to the following outage constraints:

$$
\begin{gathered}
1-\prod_{i \in\{S u b\}} \frac{1}{1+\frac{\eta_{p}}{\gamma_{p s}}\left(\frac{d_{00}}{d_{i 0}}\right)^{\alpha}} \leq \theta_{p} \\
1-\prod_{q \in\{\text { Sub } \backslash i\}} \frac{1}{1+\eta_{s}\left(\frac{d_{i j}}{d_{q j}}\right)^{\alpha}} \frac{1}{1+\eta_{s} \gamma_{p s}\left(\frac{d_{i j}}{d_{0 j}}\right)^{\alpha}} \leq \theta_{s} \\
1-\prod_{q \in\{\text { Sub } \backslash i\}} \frac{1}{1+\eta_{s}\left(\frac{d_{i j}}{d_{q j}}\right)^{\alpha}} \leq \theta_{s}
\end{gathered}
$$

where $\theta_{p}$ and $\theta_{s}$ are the maximum allowable outage probabilities of the primary and the secondary network, respectively. Note that 10 and $(11)$ correspond to the outage constraints of the first slot in which both the primary and the second systems share the spectrum band while (12) denotes the outage constraint of the secondary network in the second time slot.

From (10) we can obtain the lower bound of the power ratio $\gamma_{p s}^{l}$; from 111 we can obtain the upper bound of the power ratio $\gamma_{p s}^{u}$; and from 9 , we observe that the capacity of the secondary user decreases with the increase of the power ratio. Hence, by substituting $\gamma_{p s}^{l}$ into 9 , we achieve the following maximum average transmission capacity:

$$
C^{\prime}=\frac{1}{2} \log \left(1+\eta_{s}\right) \prod_{q \in\{\text { Sub } \backslash i\}} \frac{1}{1+\eta_{s}\left(\frac{d_{i j}}{d_{q j}}\right)^{\alpha}}\left[\frac{1}{\eta_{s} \gamma_{p s}^{l}\left(\frac{d_{i j}}{d_{0 j}}\right)^{\alpha}}+1\right]
$$

subject to the following outage constraints:

$$
\begin{gathered}
1-\prod_{q \in\{S u b \backslash i\}} \frac{1}{1+\eta_{s}\left(\frac{d_{i j}}{d_{q j}}\right)^{\alpha}} \frac{1}{1+\eta_{s} \gamma_{p s}^{l}\left(\frac{d_{i j}}{d_{0 j}}\right)^{\alpha}} \leq \theta_{s} \\
1-\prod_{q \in\{S u b \backslash i\}} \frac{1}{1+\eta_{s}\left(\frac{d_{i j}}{d_{q j}}\right)^{\alpha}} \leq \theta_{s}
\end{gathered}
$$

\section{Achievable Transmission Capacity With COOPERATIVE RELAYING}

In cooperative cognitive networks, the primary transmitter that is far away from its receiver can select a secondary user to relay its information. Assume that the distance from the PT to the relay is $d_{0 r}$, and the distance from the relay to the PR is $d_{r 0}$. We further assume that the decode-and-forward (DF) protocol is adopted by the relay. Note that the relay selected should have a higher link quality than the direct link, which indicates that:

$$
\min \left\{\frac{P_{p} \delta_{0 r} d_{0 r}^{-\alpha}}{I_{s r}}, \frac{P_{s} \delta_{r 0} d_{r 0}^{-\alpha}}{I_{s 0}}\right\}>\frac{P_{p} \delta_{00} d_{00}^{-\alpha}}{I_{s 0}}
$$

Therefore we have

$$
\left\{\begin{array}{l}
\frac{d_{0 r}^{-\alpha}}{I_{s r}}>\frac{d_{00}^{-\alpha}}{I_{s 0}} \\
d_{r 0}^{-\alpha}>\gamma_{p s} d_{00}^{-\alpha}
\end{array}\right.
$$

Then the location region of the relay can be obtained as follows:

$$
\left\{\begin{array}{l}
d_{0 r}<\left(\frac{I_{s 0}}{I_{s_{r}}}\right)^{\frac{1}{\alpha}} d_{00} \\
d_{r 0}<\gamma_{p s}^{-\frac{1}{\alpha}} d_{00}
\end{array}\right.
$$

This indicates that the location region of the relay is affected by the interference from the secondary users, the distance from the PT to the PR, as well as the power ratio. The above two equations can be illustrated by Fig. 2. When the distance $d_{00}$ between the PT and the PR is fixed, the location region of the relay, which is the shaded overlapping area of the two circles, is determined by the interference ratio $\frac{I_{s 0}}{I_{s r}}$ and the power ratio $\gamma_{p s}$. In other words, the relay selection depends on both the interference from other SUs and the transmit powers, i.e, $P_{p}$ and $P_{s}$. Moreover, the link quality and the SU capacity can not be improved if the selected relay is out of the shaded overlapping area.

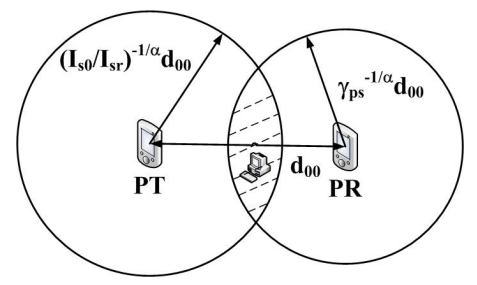

Fig. 2. The location region of the relay.

During the first time slot, the PT transmits a packet to a relay. Based on our system model, secondary users access the same spectrum as that by the PT under the constraint of the 
primary network. The successful receiving probability for the relay from the PT can be computed by:

$$
\begin{aligned}
& \operatorname{Pr}\left(S I R_{0 r} \geq \eta_{s}\right)=\operatorname{Pr}\left(\frac{P_{p} \delta_{0 r} d_{0 r}^{-\alpha}}{I_{s r}} \geq \eta_{s}\right) \\
& \quad=\operatorname{Pr}\left\{\delta_{0 r} \geq \frac{\eta_{s} d_{0 r}^{\alpha}}{P_{p}} I_{s r}\right\}=\prod_{i \in\{\text { Sub }\}} \frac{1}{1+\frac{\eta_{s}}{\gamma_{p s}}\left(\frac{d_{0 r}}{d_{i r}}\right)^{\alpha}}
\end{aligned}
$$

The successful transmission probability of a secondary user in the first slot is the same as the case without cooperative relaying, i.e.,

$$
\operatorname{Pr}_{\text {first }}^{D F}\left(S I R_{i j} \geq \eta_{s}\right)=\prod_{q \in\{S u b \backslash i\}} \frac{1}{1+\eta_{s}\left(\frac{d_{i j}}{d_{q j}}\right)^{\alpha}} \frac{1}{1+\eta_{s} \gamma_{p s}\left(\frac{d_{i j}}{d_{0 j}}\right)^{\alpha}}
$$

Thus the achievable transmission capacity of a secondary node is:

$$
C_{\text {first }}^{D F}=\log \left(1+\eta_{s}\right) \prod_{q \in\{\text { Sub } \backslash i\}} \frac{1}{1+\eta_{s}\left(\frac{d_{i j}}{d_{q j}}\right)^{\alpha}} \frac{1}{1+\eta_{s} \gamma_{p s}\left(\frac{d_{i j}}{d_{0 j}}\right)^{\alpha}}
$$

In the second slot, the relay re-encodes the message received form the PT and transmits it to the PR. Hence, the successful transmission probability for the relay to the $\mathrm{PR}$ is:

$$
\begin{aligned}
\operatorname{Pr}\left(S I R_{r 0} \geq \eta_{p}\right)= & \operatorname{Pr}\left(\frac{P_{s} \delta_{r 0} d_{r 0}^{-\alpha}+P_{p} \delta_{00} d_{00}^{-\alpha}}{I_{s 0}} \geq \eta_{p}\right) \\
= & \operatorname{Pr}\left\{\delta_{r 0} \geq \frac{\eta_{p} I_{s 0}-P_{p} d_{00}^{-\alpha} \delta_{00}}{P_{s} d_{r 0}^{-\alpha}}\right\} \\
= & \mathrm{E}_{\left\{\delta_{i 0}\right\}} \exp \left(-\frac{\eta_{p} d_{r 0}^{\alpha}}{P_{s}} I_{s 0}\right) \\
& \times \mathrm{E}_{\left\{\delta_{00}\right\}} \exp \left(\frac{P_{p} d_{r 0}^{\alpha} \delta_{00}}{P_{s} d_{00}^{\alpha}}\right) \\
= & \prod_{i \in\{S u b\}} \frac{1}{1+\eta_{p}\left(\frac{d_{r 0}}{d_{i 0}}\right)^{\alpha}} \frac{1}{1-\gamma_{p s}\left(\frac{d_{r 0}}{d 00}\right)^{\alpha}}
\end{aligned}
$$

The successful transmission probability for a secondary user $i$ to its (secondary) destination $j$ is:

$$
\begin{aligned}
\operatorname{Pr}_{\text {second }}^{D F} & \left(S I R_{i j} \geq \eta_{s}\right)=\operatorname{Pr}\left(\frac{P_{s} \delta_{i j} d_{i j}^{-\alpha}}{I_{s j}+I_{r j}} \geq \eta_{s}\right) \\
= & \exp \left(-\frac{\eta_{s} d_{i j}^{\alpha}}{P_{s}}\left(I_{s j}+I_{r j}\right)\right) \\
= & \mathrm{E}_{\left\{\delta_{q j}\right\}}\left\{\exp \left(-\frac{\eta_{s} d_{i j}^{\alpha}}{P_{s}} \sum_{q \in\{S u b \backslash i\}} P_{s} \delta_{q j} d_{q j}^{-\alpha}\right)\right\} \\
& \times \mathrm{E}_{\left\{\delta_{r j}\right\}} \exp \left(-\frac{\eta_{s} d_{i j}^{\alpha}}{P_{s}} P_{s} d_{r j}^{-\alpha} \delta_{r j}\right) \\
= & \prod_{q \in\{S u b \backslash i\}} \frac{1}{1+\eta_{s}\left(\frac{d_{i j}}{d_{q j}}\right)^{\alpha}} \frac{1}{1+\eta_{s}\left(\frac{d_{i j}}{d_{r j}}\right)^{\alpha}}
\end{aligned}
$$

Then the achievable transmission capacity of a secondary node can be given by:

$$
C_{\text {second }}^{D F}=\log \left(1+\eta_{s}\right) \prod_{q \in\{\text { Sub } \backslash i\}} \frac{1}{1+\eta_{s}\left(\frac{d_{i j}}{d_{q j}}\right)^{\alpha}} \frac{1}{1+\eta_{s}\left(\frac{d_{i j}}{d_{r j}}\right)^{\alpha}}
$$

The outage probability for the transmission from the PT to the PR is $1-\operatorname{Pr}\left(S I R_{0 r} \geq \eta_{s}\right) \operatorname{Pr}\left(S I R_{r 0} \geq \eta_{p}\right)$. Hence, from (19) 24), the average transmission capacity of a secondary user with outage constraints from both the primary and the secondary network can be derived as follows:

$$
\begin{aligned}
C^{D F}= & \frac{1}{2}\left(C_{\text {first }}^{D F}+C_{\text {second }}^{D F}\right) \\
= & \frac{1}{2} \log \left(1+\eta_{s}\right) \prod_{q \in\{\text { Sub } \backslash i\}} \frac{1}{1+\eta_{s}\left(\frac{d_{i j}}{d_{q j}}\right)^{\alpha}}\left[\frac{1}{1+\eta_{s} \gamma_{p s}\left(\frac{d_{i j}}{d_{0 j}}\right)^{\alpha}}\right. \\
& \left.+\frac{1}{1+\eta_{s}\left(\frac{d_{i j}}{d_{r j}}\right)^{\alpha}}\right]
\end{aligned}
$$

subject to the following outage constraints:

$$
\begin{array}{r}
1-\prod_{i \in\{\text { Sub }\}} \frac{1}{1+\frac{\eta_{s}}{\gamma_{p s}}\left(\frac{d_{0 r}}{d_{i r}}\right)^{\alpha}} \frac{1}{1+\eta_{p}\left(\frac{d_{r 0}}{d_{i 0}}\right)^{\alpha}} \frac{1}{1-\gamma_{p s}\left(\frac{d_{r 0}}{d 00}\right)^{\alpha}} \leq \theta_{p} \\
1-\prod_{q \in\{S u b \backslash i\}} \frac{1}{1+\eta_{s}\left(\frac{d_{i j}}{d_{q j}}\right)^{\alpha}} \frac{1}{1+\eta_{s} \gamma_{p s}\left(\frac{d_{i j}}{d_{0 j}}\right)^{\alpha}} \leq \theta_{s} \\
1-\prod_{q \in\{\text { Sub } \backslash i\}} \frac{1}{1+\eta_{s}\left(\frac{d_{i j}}{d_{q j}}\right)^{\alpha}} \frac{1}{1+\eta_{s}\left(\frac{d_{i j}}{d_{r j}}\right)^{\alpha}} \leq \theta_{p}
\end{array}
$$

From 26 and 27, we can obtain the lower bound $\gamma_{p s}^{l}$ and the upper bound $\gamma_{p s}^{u}$ of the power ratio, respectively. We also observe that the lower bound $\gamma_{p s}^{l}$ is affected by the location of the relay. From (25), we further observe that the capacity of the secondary user decreases with the increase of the power ratio. Hence by substituting $\gamma_{p s}^{l}$ into 25 , we obtain the following maximum transmission capacity $C^{*}$ of the cooperative cognitive network:

$$
\begin{aligned}
C^{*}= & \frac{1}{2} \log \left(1+\eta_{s}\right) \prod_{q \in\{S u b \backslash i\}} \frac{1}{1+\eta_{s}\left(\frac{d_{i j}}{d_{q j}}\right)^{\alpha}}\left[\frac{1}{1+\eta_{s} \gamma_{p s}^{l}\left(\frac{d_{i j}}{d_{0 j}}\right)^{\alpha}}\right. \\
& \left.+\frac{1}{1+\eta_{s}\left(\frac{d_{i j}}{d_{r j}}\right)^{\alpha}}\right]
\end{aligned}
$$

subject to the following constraints:

$$
\begin{gathered}
1-\prod_{q \in\{S u b \backslash i\}} \frac{1}{1+\eta_{s}\left(\frac{d_{i j}}{d_{q j}}\right)^{\alpha}} \frac{1}{1+\eta_{s} \gamma_{p s}^{l}\left(\frac{d_{i j}}{d_{0 j}}\right)^{\alpha}} \leq \theta_{s} \\
1-\prod_{q \in\{S u b \backslash i\}} \frac{1}{1+\eta_{s}\left(\frac{d_{i j}}{d_{q j}}\right)^{\alpha}} \frac{1}{1+\eta_{s}\left(\frac{d_{i j}}{d_{r j}}\right)^{\alpha}} \leq \theta_{p}
\end{gathered}
$$

\section{Numerical ANALYSis}

In this section, we report our numerical results on the average achievable transmission capacity of the cognitive radio network with or without cooperative relaying based on our analysis. For simplicity, we consider a simple network topology shown in Fig. 3, where the relay is located in the straight line between the PT and the PR, and the two sources of the secondary network have the same distance to the PR. As elaborated in the following subsections, such a simple topology can perfectly capture the insights of our analysis while facilitating the thorough comprehension of the numerical 


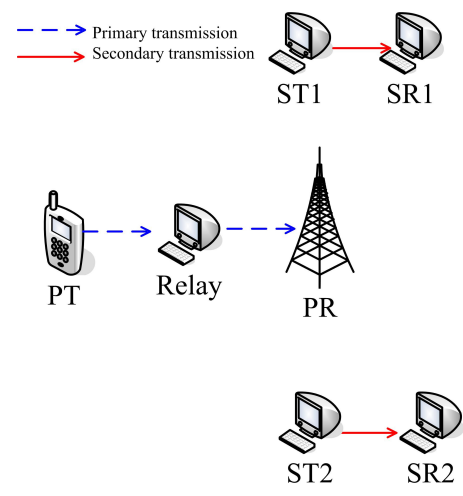

Fig. 3. The topology utilized for the numerical analysis.

TABLE I

The Simulation Parameter Settings

\begin{tabular}{|c|l|c|}
\hline symbol & Semantic Meaning & Value \\
\hline$\alpha$ & pass loss exponent & 4 \\
\hline$\eta_{p}$ & $\begin{array}{l}\text { threshold of the PR in the } \\
\text { primary network }\end{array}$ & $4 \mathrm{~dB}$ \\
\hline$d_{00}$ & distance between PT and PR & $100 \mathrm{~m}$ \\
\hline$d_{i 0}$ & $\begin{array}{l}\text { distance between a secondary } \\
\text { transmitter and the PR }\end{array}$ & $100 \mathrm{~m}$ \\
\hline$d_{i j}$ & $\begin{array}{l}\text { distance between a secondary } \\
\text { transmitter and its destination }\end{array}$ & $20 \mathrm{~m}$ \\
\hline$d_{0 j}$ & $\begin{array}{l}\text { distance between the PT } \\
\text { and a secondary receiver }\end{array}$ & $\sqrt{120^{2}+100^{2}} \mathrm{~m}$ \\
\hline$d_{r 0}$ & \begin{tabular}{l} 
distance between relay and PR \\
\hline$d_{0 r}$
\end{tabular} & distance between PT and relay \\
\hline$d_{i r}$ & $\begin{array}{l}\text { distance between the secondary } \\
\text { transmitter and the relay }\end{array}$ & $\sqrt{100^{2}+100^{2}} \mathrm{~m}$ \\
\hline$d_{r j}$ & $\begin{array}{l}\text { distance between the relay } \\
\text { and a secondary receiver }\end{array}$ & $\sqrt{100^{2}+(120)^{2}} \mathrm{~m}$ \\
\hline
\end{tabular}

results. The distance calculation as well as other parameter settings utilized in our simulation study are listed in Table I.

First we consider the case when the receiving threshold of the secondary network is set to be the same as that of the PR $(4 \mathrm{~dB})$. The achievable transmission capacity of the cognitive network versus the distance from the relay to the primary receiver when the power ratio is set to be 5,10 , or 50 , is shown in Fig. 4. From the figure we observe that without cooperative

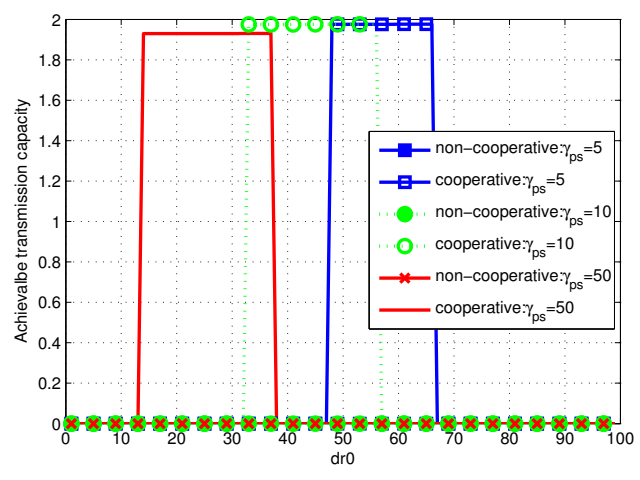

Fig. 4. The achievable transmission capacity of the cognitive network versus $d_{r 0}$ when the power ratio varies. relaying the network can not satisfy the outage probability constraints from the primary or the secondary system for all parameter settings, thus achieving a zero capacity. When the power ratio is set to 5 , and the distance from the relay to the PR changes from $48 \mathrm{~m}$ to $68 \mathrm{~m}$ (this distance range defines the location region of the relay), the network with cooperative relaying can satisfy the outage probability constraints and achieve a capacity value of around 2 . When the power ratio changes, the feasible location of the relay also changes, which is consistent with our previous analysis in section IV]

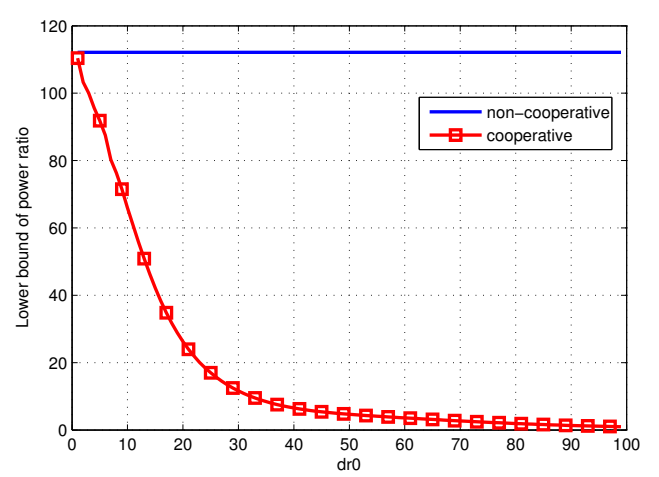

Fig. 5. The lower bound of the power ratio versus $d_{r 0}$.

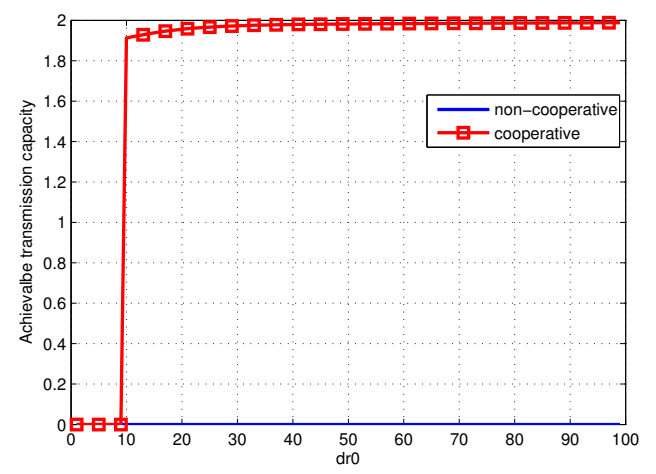

Fig. 6. The maximum achievable transmission capacity of the cognitive network versus $d_{r 0}$.

Since the transmission capacity decreases with the increase of the power ratio, we also investigate the lower bound of the power ratio in this simulation study. Fig. 5 reports the lower bound of the power ratio (computed from $(26)$ ) versus the distance from the relay to the primary receiver. For comparison purpose we also draw the lower bound of the power ratio computed from (10) when no cooperative relaying is adopted. From this figure we observe that the lower bound of the power ratio decreases with the increase of the distance from the relay to the PR. This is because when the relay is nearer to the PR, the PR can experience a lower pass loss such that the secondary user can increase its transmission power for capacity enhancement. The maximum achievable transmission capacity when adopting the lower bound of the power ratio is given in 
Fig. 6 Since the outage probability increases with the power ratio, the maximum capacity is only achieved when $d_{r 0}$ is higher than 10 under which the outage probability constraints are satisfied, according to 30 .

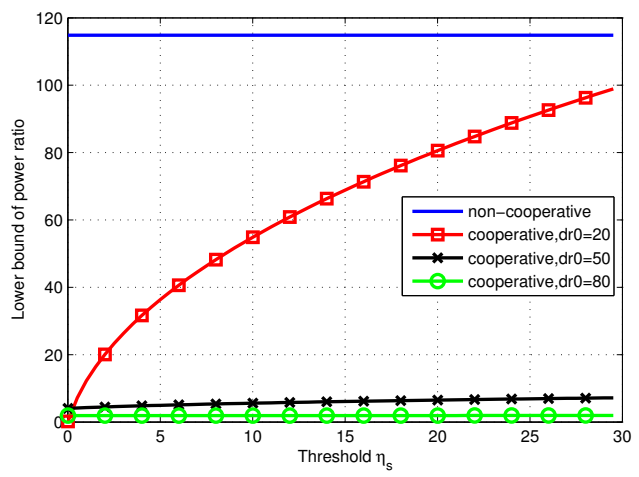

Fig. 7. The lower bound of the power ratio versus the threshold $\eta_{s}$

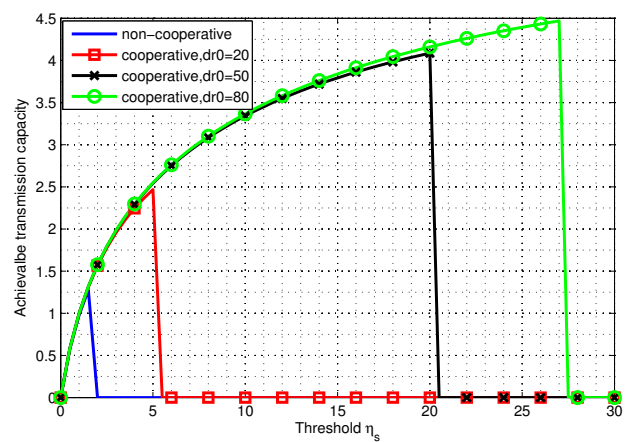

Fig. 8. The maximum achievable transmission capacity of the cognitive network versus the threshold $\eta_{s}$.

The lower bound of the power ratio and the maximum average transmission capacity of the network with/without cooperative relaying versus the receiving threshold when the relay is fixed to $20 \mathrm{~m}, 50 \mathrm{~m}$, or $80 \mathrm{~m}$ away from the PR are shown in Fig. 7 and Fig. 8, respectively. We observe that under the outage probability constraints of both the primary and the secondary system the maximum receiving threshold of the secondary network is $2 \mathrm{~dB}$ without cooperative relaying while it reaches 5, 20, and 27 with cooperative relaying when the relay is respectively $20 \mathrm{~m}, 50 \mathrm{~m}$, and $80 \mathrm{~m}$ away from the PR. This can be explained as follows: When the relay is nearer to the PR, the PR can experience a lower pass loss such that the secondary user can increase its transmission power. Accordingly the secondary receiver can achieve a higher SIR when the power ratio between the primary and the secondary network decreases. Thus the secondary user can receive its signals successfully with a higher threshold. Given a higher threshold $\eta_{s}$, the network with cooperative relaying can achieve a larger capacity than the one without cooperative relaying.

\section{CONCLUSION}

In this paper, we investigate the average achievable transmission capacity of a cognitive network that provides cooperative relaying to the primary network under the outage probability constraints from both the primary and the secondary system. The probabilities of successful transmissions in the primary and the secondary network are respectively derived for the direct transmission and the decode-and-forward relay model. The maximum achievable transmission capacities of the secondary network with or without cooperative relaying in terms of bits $/ \mathrm{hop} / \mathrm{s} / \mathrm{Hz} /$ node are obtained based on Shannon's Theory. Our numerical results indicate that cooperative relaying between the primary and the secondary network can help the secondary network to achieve a higher transmission capacity when the relay is located at an appropriate position. For future research, we will consider more complicated cooperative cognitive network scenarios and investigate an efficient relay selection algorithm.

\section{ACKNOWLEDGMENT}

The authors would like to thank the support from the National Natural Science Foundation of China (Grants No.61073160 and No.61172074) and the National Science Foundation of the US (CNS-0831852).

\section{REFERENCES}

[1] T. jing, X. Chen, H. Huo and X. cheng, "Achievable Transmission Capacity of Cognitive Mesh Networks With Different Media Access Control," IEEE INFOCOM 2012, pp. 1764-1772.

[2] G. D. Zhao, C. Y. Yang, G. Y. Li, D. D. Li, and A. C. K. Soong, "Power and channel allocation for cooperative relay in cognitive radio networks," IEEE Journal on Selected Topics in Signal Processing, vol. 5, no. 1, pp. 151-159, Feb. 2011.

[3] J. Jia, J. Zhang, and Q. Zhang, "Cooperative relay for cognitive radio networks," IEEE INFOCOM 2009, pp. 2304-2312.

[4] O. Simeone, U. Spagnolini, and Y. Bar-Ness, "Stable throughput of cognitive radios with and without relaying capability," IEEE Trans. Commun., vol. 55, no. 12, pp. 2351-2360, Dec. 2007.

[5] S. Kompella, G. D. Nguyen, J. E. Wieselthier and A. Ephremides, "Stable throughput tradeoffs in cognitive shared channels with cooperative relaying ," IEEE INFOCOM 2011, pp. 1961-1969.

[6] J. Zhang and Q. Zhang, "Stackelberg game for utility-based cooperative cognitiveradio networks," ACM MobiHoc 2009, pp. 23-32.

[7] K. Hong and Y. Hua, "Throughput analysis of large wireless networks with regular topologies," EURASIP Journal on Wireless Comm Net, 2007, Article ID 26760, 11 pages.

[8] S. Jeon, N. Devroye, M. Vu, S. Chung, and V. Tarokh, "Cognitive networks achieve throughput scaling of a homogeneous network," IEEE Trans. Info. Theory, vol. 57, no. 8, pp. 5103-5115, Aug. 2011.

[9] J. Lee, S. Lim, J. G. Andrews, and D. Hong, "Achievable transmission capacity of secondary system in cognitive radio networks," IEEE ICC, 2010.

[10] S. Weber, X. Yang, J. G. Andrews, and G. de. Veciana, "Transmission capacity of wireless ad hoc networks with outage constraint," IEEE Trans. Info. theory, vol. 51, no. 12, pp. 4091-4102, Dec. 2005.

[11] K. Huang, V. K. N. Lau, and Y. Chen, "Spectrum sharing between cellular and mobile ad hoc networks: Transmission-Capacity Trade-Off,' IEEE Journal on Selected Areas in Communications, vol. 27, no. 2, pp. 1256-1267, Aug. 2008.

[12] C. Li and H. Dai, "Transport throughput of secondary networks in spectrum sharing systems," IEEE INFOCOM 2011, pp. 2732-2740.

[13] W. Huang and X. Wang, "Throughput and delay scaling of general cognitive networks," INFOCOM 2011, pp. 2210-2218. 